\title{
Tyrosine hydroxylase immunoreactivity identifies possible catecholaminergic fibers in the organ of Corti
}

\author{
Nancy Jones ', Jörgen Fex ${ }^{1}$ and Richard A. Altschuler ${ }^{2}$ \\ 'Laboratory of Neuro-otolaryngology, NINCDS, N.I.H., Bethesda, U.S.A. \\ and ${ }^{2}$ Kresge Hearing Research Institute, University of Michigan, Ann Arhor, U.S.A.
}

(Received 28 January 1987: accepted 25 April 1987)

\begin{abstract}
Antibodies to tyrosine hydroxylase, dopamine $\beta$-hydroxylase and phenylethanolamine $N$-methyltransferase were used in an immunocytochemical examination of catecholamines in the cochlea. In cryostat sections. tyrosine hydroxylase and dopamine $\beta$-hydroxylase-like immunoreactivities fibers were seen in the modiolus that did not extend to the organ of Corti. These corresponded to blood vessel associated and non blood vessel associated fibers that have been previously described with histofluorescence. In surface preparations, tyrosine hydroxylase-like immunoreactivity was seen in the organ of Corti. in the inner and lunnel spiral bundles, suggesting an efferent component may be catecholaminergic.

Catecholamine; Cochlea; Transmitter; Efferent fiber; Immunocytochemistry
\end{abstract}

\section{Introduction}

In the 1940's and 1950's attempts were made to treat patients with diseases of the inner ear through manipulation of the autonomic innervation of the cochlea and animal studies were initiated to provide a theoretical basis for such treatment (Beickert et al., 1956). Seymour and Tappan (1951) described a relationship between cervical sympathetic stimulation and cochlear microphonics, but Krejci and Bornschein (1954) found no such effect. Such contradictory results of early studies are described by Beickert et al. (1956). In more recent studies Baust et al. (1964) and Pickles (1979) found sympathetic stimulation had no effect on cochlear microphonics, but an effect of sympathetic stimulation on compound action potential (CAP), however, was reported by Pickles (1979). Hultcrantz et al. (1982) found a $30 \%$ decrease of amplitude of evoked CAP after a cervical sympathectomy in guinea pig and Muchnik et al. (1983) found injection of catecholamines into

Correspondence to: R.A. Altschuler, Kresge Hearing Research Institute. Iniversity of Michigan. Ann Arbor, MI 48109. U.S.A. the maxillary artery increased the mean threshold for CAP. Lee and Møller (1985) have recently shown that stimulation of the superior cervical ganglion resulted in an increase in the $N_{1}$ latency of the CAP equivalent to a decrease in sensitivity of approximately $5 \mathrm{~dB}$ near threshold, but the amplitude of this component showed no systematical change related to sympathetic stimulation. Sympathetics also influence cochlear blood flow (Suga and Snow, 1969; Hultcrantz et al.. 1977), as shown recently through laser doppler analysis (Hultcrantz et al., 1986). Cervical sympathetectomy also results in an increase in perilymphatic oxygen tension (Nuttall. Pers. Commun.).

The development of the Falck-Hillarp technique (Carlsson et al., 1962: Falck. 1962; Dahlström and Fuxe, 1964) of catecholamine fluo-rescence allowed for a reliable and specific detection of catecholaminergic fibers. With the use of this method, two different groups of adrenergic fibers were found in the mammalian cochlea: one blood vessel dependent, found only around the labyrinthine artery and its greater branches; the other a blood vessel independent system of un- 
myelinated fibers traveling peripherally with the cochlear nerve (Fex et al., 1965; Spoendlin and Lichtensteiger, 1966; Terayama et al., 1966; Vinnikov et al., 1966; Ross, 1971; Densert, 1974; Densert and Flock, 1974). This second system was located in the osseous spiral lamina and formed a dense terminal plexus around the habenula perforata but did not extend more peripherally. Vinnikov et al. (1966) also reported the presence of a separate group of fibers, an efferent adrenergic system that existed beyond the habenula and ran in the inner spiral bundle, however, an earlier study (Fex et al., 1965) and all subsequent studies, reviewed by Densert (Densert, 1974; Densert and Flock, 1974) did not find positive fluorescence in this area. Vinnikov et al. (1966) used cochleae prepared in a special manner, described as isolated both in dry form and physiological solution (Vinnikov et al., 1966), which may have been optimal for applying the Falck-Hillarp method to the cochlea.

With the availability of antibodies to enzyme markers (antibodies to catecholamine biosynthetic enzymes; Eugene Tech. International, Inc, Allendale, NJ) for catecholamines, tyrosine hydroxylase (TH), dopamine $\beta$-hydroxylase (DPH) and phenylethanolamine $N$-methyl transferase (PNMT) that can be used for immunocytochemistry (Pickel et al., 1975), we have re-examined the adrenergic innervation of the guinea pig cochlea in immunocytochemical studies. The enzymes each catalyze different steps in the production of catecholamines (Levitt et al., 1965) and depending on which are localized, the presence of a particular catecholamine or catecholamines are suggested. If only the TH immunoreactive label is seen, this suggests the presence of dopamine, if $\mathrm{TH}$ and DPH immunoreactivities are seen, then the presence of norepinephrine is suggested without excluding dopamine, and if TH, DPH and PNMT immunoreactivities are all seen, then epinephrine is suggested without excluding dopamine or norepinephrine.

\section{Materials and Methods}

Twelve female NIH strain guinea pigs, $150-200$ $\mathrm{g}$, were used. Animals were anesthetized with chloral hydrate and perfused through the heart with
$0.1 \mathrm{M}$ cacodylate buffer at $\mathrm{pH} 7.3$ followed by a 4\% paraformaldehyde fixative in the same buffer. Cochleae were removed, perfused locally through the round window with the fixative and then immersed for $1 \mathrm{~h}$ in the fixative. Bony shell, stria vascularis and tectorial membrane were then removed, as described previously (Fex and Altschuler, 1985). Nine cochleae, from 6 animals, were processed for immunofluorescence, using the method of Coons et al. (1942) as described previously (Fex and Altschuler, 1985). Briefly, they were placed in $2 \%$ EDTA and $6 \%$ sucrose in phosphate buffered saline (PBS) at $\mathrm{pH} 7.2$ for $16-$ $40 \mathrm{~h}$ at $4^{\circ} \mathrm{C}$, with several changes of fluids. Cochleae were then frozen on cryostat chucks and $10 \mu \mathrm{m}$ sections were cut on a cryostat. Sections were incubated overnight in antiserum to $\mathrm{TH}$. DBH or PNMT, diluted $1: 1000-5000$, for $16-20$ $\mathrm{h}$, at $4^{\circ} \mathrm{C}$. Sections were then rinsed in PBS, incubated with FITC-labeled goat anti-rabbit IgG that had been diluted $1: 10$ in PBS, for $30 \mathrm{~min}$, at $37^{\circ} \mathrm{C}$, rinsed in PBS, coverslipped and examined under epifluorescent illumination. Fifteen cochleae from 9 animals were used for immunoperoxidase staining. Cochleae were rinsed for $16-20$ h at $4^{\circ} \mathrm{C}$ in PBS and then placed for $1 \mathrm{~h}$ in $10 \%$ normal goat serum in PBS. The cochlear spirals were then incubated in antiserum to either TH, DBH or PNMT that had been diluted $1: 1000-5000$ in PBS with $0.3 \%$ Triton $\mathrm{X}-100$. The avidin-biotin peroxidase complex $(A B C)$ staining method of Hsu et al. (1981) was followed using the protocol for the Vectastain (Vector Laboratories) kit. Diaminobenzidine was used as a chromogen, as described previously (Fex and Altschuler, 1985). Segments of the cochlear spiral were removed, as described previously (Fex and Altschuler, 1985), and viewed under bright-field and differential interference contrast optics on a Zeiss Universal or Leitz Orthomat photomicroscope.

The brainstems were removed from several animals, immersed for $1 \mathrm{~h}$ in fixative, rinsed overnight in PBS and sectioned in PBS with a vibratome. Immunoperoxidase staining using antisera to TH and DPH was performed on $50 \mu \mathrm{m}$ sections through the lateral superior olivary complex using the Vectastain kit protocol and the $\mathrm{ABC}$ technique as described above and previously (Altschuler et al., 1984). 

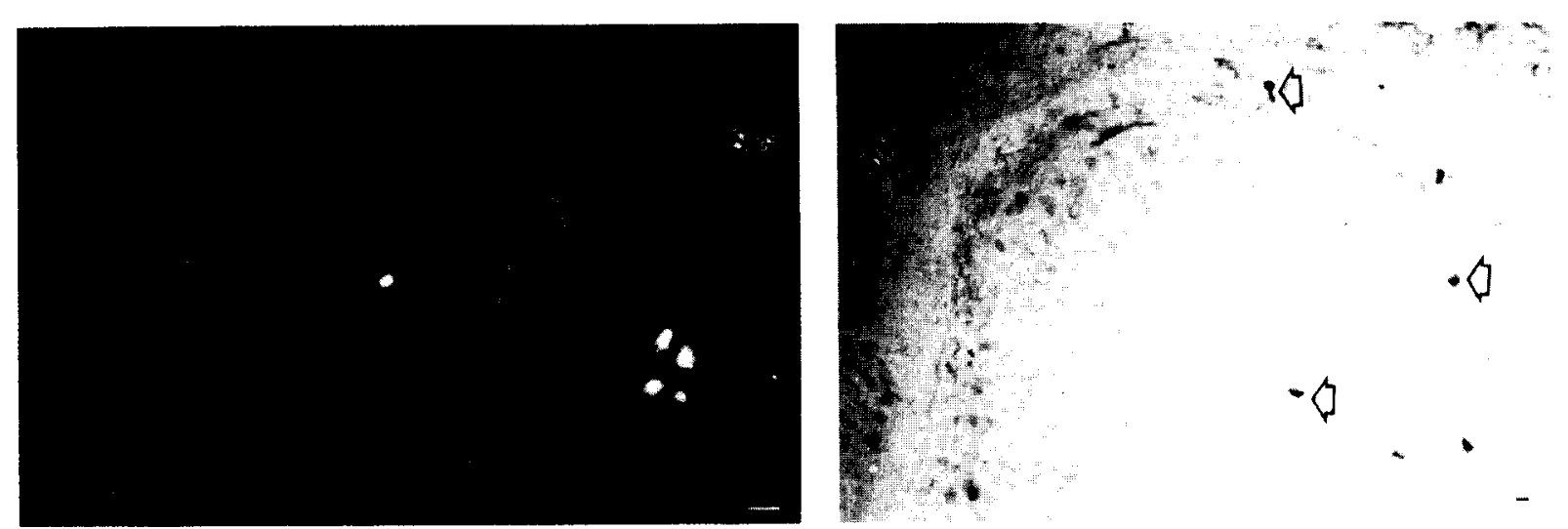

-
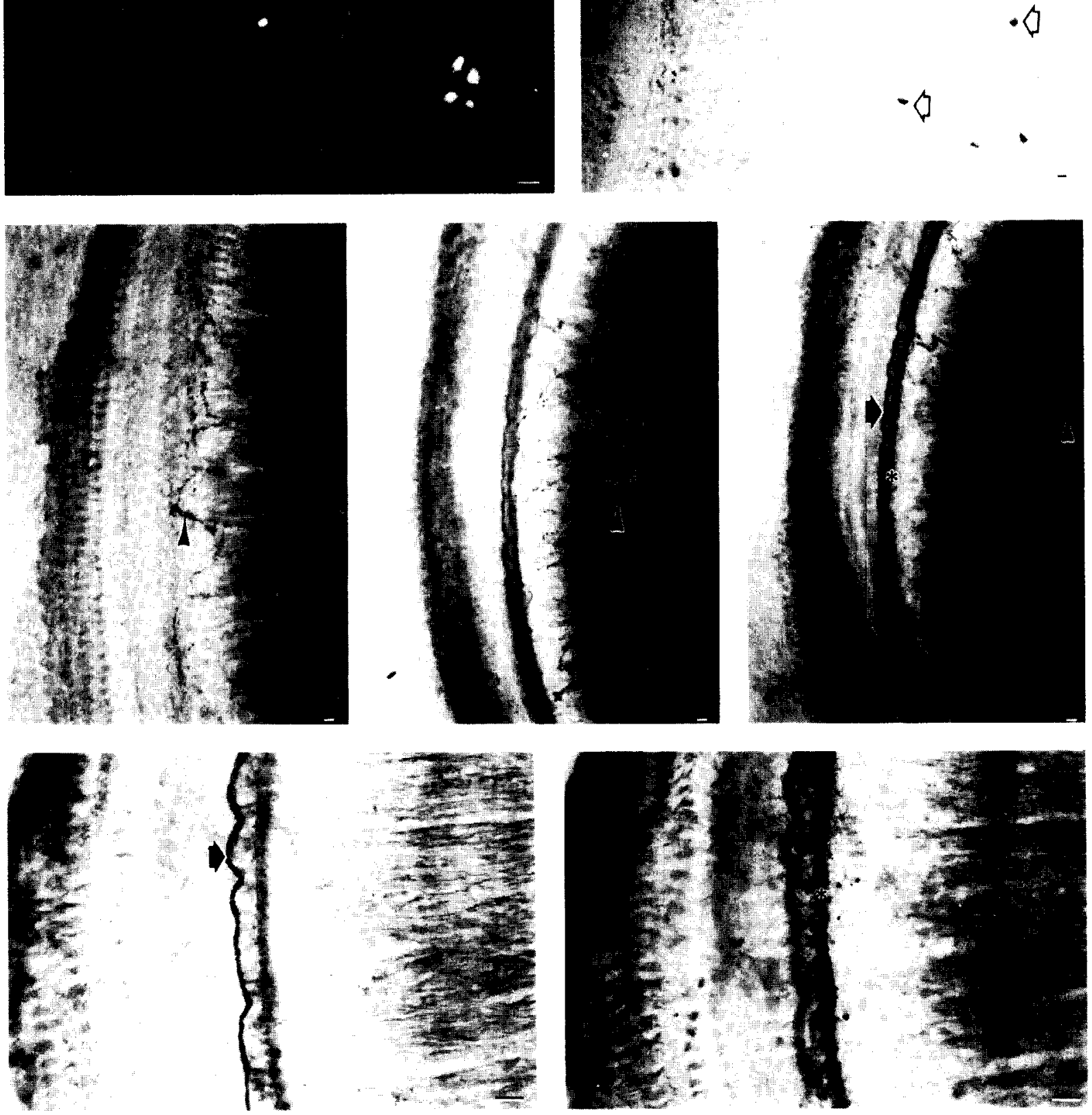

Fig. 1. Tyrosine hydroxylase (TH) and dopamine $\beta$-hydroxylase (DPH) immunoreactivities with immunofluorescent (a) and immunoperoxidase (b-g) techniques. (a) TH-like immunoreactivity in a cryostat section through the modiolus (with the organ of Corti out of the figure towards the left). Arrowheads point to immunofluorescent fibers. SG, spiral ganglion cells. (b) TH-like immunoreactive cells (three of which are marked by open arrows) in a vibratome section through the lateral superior olive. (c) DPH-like immunoreactive fibers (arrowhead) associated with blood vessels in a surface preparation from the second turn of the guinea pig cochlea. (d-g) TH-like immunoreactive fibers in surface preparations from the second turn of guinea pig cochleae. Blood vessel-associated fibers (arrowheads) plus fibers in the tunnel spiral bundle (dark arrows) and inner spiral bundle (asterix) are seen. 


\section{Results}

\section{Immunofluorescence}

TH- and DBH-like immunoreactivities, but not PNMT-like immunoreactivity, were seen in cryostat sections through the guinea pig cochlea in fibers with distributions similar to those previously described as adrenergic on the basis of histofluorescence (Fex et al., 1965; Spoendlin and Lichtensteiger, 1966; Terayama et al., 1966; Vinnikov et al., 1966; Ross, 1971; Densert, 1974; Densert and Flock, 1974), electron microscopic (Ross, 1971; Densert and Flock, 1974) and autoradiographic (Eybalin et al., 1983) techniques. THlike (Fig. 1a) and DPH-like immunoreactive staining were observed on adjacent sections and had similar patterns of distribution, suggesting these were present in the same fibers. Two sets of fibers were seen, one was associated with blood vessels and the other was not. These fibers were seen in the modiolus, extending from the region of spiral ganglion cells to the tympanic lip and not extending into the organ of Corti. A weak diffuse fluorescence was seen by the inner hair cell bases in the organ of Corti with antiserum to $\mathrm{TH}$ and not seen with antiserum to DBH and to PNMT. This labeling, however, was not sufficient to provide positive identification.

\section{Immunoperoxidase}

TH-like and DPH-like immunoreactivity, but not PNMT-like immunoreactivity, were seen with immunoperoxidase staining on surface preparations of segments of the cochlear spiral. Medial to the tympanic lip, TH-like and DPH-like immunoreactivity were seen in fibers associated with blood vessels (Fig. 1c-e). This was the only DPHlike immunoreactivity found (Fig. 1c). TH-like immunoreactivity was also found in fibers in the inner spiral bundle and tunnel spiral bundle, throughout their entire length (Fig. $1 \mathrm{~d}-\mathrm{g}$ ). No communication was observed between these two sets of TH-like immunoreactive fihers. The TH-like immunoreactivity was greater in the tunnel spiral bundle than in the inner spiral bundel (Fig. 1d-g), in contrast to other immunoreactive labelings of these systems we have previously observed with antisera to other neurotransmitter candidates; enkephalins, dynorphins, ChAT-a marker for acetyl- choline (Fex and Altschuler, 1985; Altschuler and Fex, 1986; Altschuler et al., 1986b). To confirm this labeling in fibers recently classified as lateral olivocochlear efferents (Guinan et al., 1983: Warr et al., 1986) we examined their cells which originated from the lateral superior olivary complex, and which we have previously shown to be immunoreactive for choline acetyltransferase (ChAT), enkephalins and dynorphins (Altschuler et al., 1984, 1986b; Fex and Altschuler, 1985). When immunoperoxidase immunocytochemistry, using antibodies to TH and DPH as described above, was performed on vibratome sections through the auditory brainstem, TH-like immunoreactive cells with the morphology and distribution of lateral olivocochlear neurons were found in the lateral superior olivary complex (Fig. 1b), while no DPH-like immunoreactivity was observed in this area. This result in guinea pig (Altschuler et al., 1986a) has recently been confirmed (Vincent, 1986) in the hamster.

\section{Discussion}

The results of this study provide immunocytochemical verification of the histofluorescence studies of catecholamines in the cochlea. The presence of TH-like and DPH-like immunoreactivities, but not PNMT immunoreactivity, in blood vesseland non-blood vessel-associated fibers in the guinea pig modiolus suggest that noradrenaline (norepinephrine) may serve as a transmitter in these fibers, but does not preclude dopamine also being present in these fibers. While recent physiological studies suggest that these fibers effect the compound action potential (Baust et al., 1964; Pickles, 1979; Hultcrantz et al., 1982; Lee and Møller, 1985) with a delay in $N_{1}$ response (Lee and Møller, 1985) and also cause a change in cochlear blood flow (Suga and Snow, 1969; Hultcrantz et al., 1977, 1986) the mechanism by which they may achieve these effects is not clear.

The presence of TH-like immunoreactivity, but not DPH- or PNMT-like immunoreactivities, in olivocochlear fibers in the cochlea and olivocochlear neurons in the lateral superior olivary complex would suggest that dopamine may be added to the long list of lateral olivocochlear neurotransmitter candidates (for review see Fex 
and Altschuler, 1985; Altschuler and Fex, 1986; Altschuler et al., 1986b; Klinke, 1986). Bobbin and Thompson (1978) found that intracochlear application of dopamine had no effect on CAP or cochlear microphonics. Klinke and Evans (1977) have shown that the intracochlear application of a mixture of adrenalin and noradrenaline has no effect on CAP or single fiber activity, however this does not preclude dopamine having some efferent function. Although the finding of TH-like immunoreactivity in cochlear efferents is consistent with the early findings of Vinnikov (1966), it is not consistent with other reports using histofluorescence (Fex et al., 1965; Spoendlin and Lichtensteiger, 1966; Terayama et al., 1966; Vinnikov, 1966; Ross, 1971; Densert, 1974; Densert and Flock, 1974) electron microscopic (Ross, 1971; Densert and Flock, 1974) and autoradiographic (Eybalin et al., 1983) techniques. It should be noted that the antibody to $\mathrm{TH}$ might cross-react with another substance with antigenic sites in common with TH. Gil-Loyzaga and Pares-Herbute (1986) recently used high performance liquid chromatography to show both dopamine and noradrenaline in the cochlea, but did not distinguish between modiolus and organ of Corti. Further studies, e.g., using antisera directly to dopamine will be needed to confirm our findings suggesting dopamine is present in lateral olivocochlear efferents where it may function as a neurotransmitter/ modulator.

\section{References}

Altschuler, R.A. and Fex, J. (1986) Efferent neurotransmitters. In: Neurobiology of Hearing: The Cochlea, pp. 383-396. Editors: Altschuler, R.A., Hoffman, D.W. and Bobbin, R.P.. Raven Press, NY.

Altschuler, R.A., Fex, J., Parakkal, M.H. and Eckenstein, F. (1984) Colocalization of enkephalin-like and choline acetyltransferase-like immunoreactivities in olivocochlear neurons of the guinea pig. J. Histochem. Cytochem. 32, $839-843$

Altschuler. R.A., Jones, N., Reeks, K.A. and Fex, J. (1986a) Tyrosine hydroxlase immunoreactivity marks a catecholaminergic system in the guinea pig organ of Corti. Abstr. Assoc. Res. Otolaryngol., p. 91.

Altschuler. R.A., Hoffman, D.W. and Wenthold, R.J. (1986b) Neurotransmitters of the cochlea and cochlear nucleus: Immunocytochemical evidence. Am. J. Otolaryngol. 7. $100-106$

Baust. W.. Berlucchi, G. and Moruzzi, G. (1964) Changes in the auditory input during arousal in cats with tenotomized middle ear muscles. Arch. Ital. Biol. 102, 675-685.

Beickert, P., Gisselsson, L. and Löfström, B., (1956) Der einfluss des sympatischen nervensystems auf das innerohr. Arch. Ohren Nasen Kehlkopfheilkd. 168, 495-507.

Bobbin, R.P. and Thompson, M.H. (1978) Effects of putative transmitters on afferent cochlear transmission. Ann. Otol. Rhinol. Larngol. 91. 209-215.

Carlsson, A., Falck, B. and Hillarp, N.-A. (1962) Cellular localization of brain monoamines. Acta. Physiol. Scand. Suppl. 56, 196-215.

Coons, A.H., Creech, H.J., Jones, R.N. and Berliner, E. (1942) The demonstration of pneumococcal antigen in tissue by the use of fluorescent antibody. J. Immunol. 45, 159-170.

Dahlström, A. and Fuxe, K. (1964) A method for the demonstration of monoamine-containing nerve fibers in the central nervous system. Acta Physiol. Scand. 60. 293-295.

Densert, O. (1974) Adrenergic innervation in the rabbit cochlea. Acta Otu-Laryngul. 78, 345-356.

Densert. O. and Flock. A. (1974) An electron microscopic study of adrenergic innervation in the cochlea. Acta OtoLaryngol. 77. 185-197.

Eybalin, M., Calas, A. and Pujol, R. (1983) Radioautographic study of the sympathetic fibers of the cochlea. Acta OtoLaryngol. 96. 69-74.

Falck, B. (1962) Observations on the possibilities of the cellular localization of monoamines by a fluorescent method. Acta Physiol. Scand. 56 (Suppl. 197), 1-25.

Fex. J. and Altschuler, R.A.. (1985) Immunocytochemistry of the mammalian cochlea: Results and expectations. In: Auditory Biochemistry, pp. 5-28. Fditor: D.G. Drescher. Charles Thomas, Springfield, IL.

Fex, J., Fuxe, K. and Lennerstrand. G. (1965) Absence of Monoamines in olivo-cochlear fibres in cat. Acta Physiol. Scand. 64, 259-262.

Gil-Loyzaga. P. and Pares-Herbute. N. (1986) Presence of noradrenaline and dopamine in the adult and developing rat cochlea. Neurosci. Lett. Suppl. 26, S134.

Guinan, J.J., Jr., Warr, W.B. and Norris, B.E. (1983) Differential olivocochlear projections from lateral versus medial zones of the superior olivary complex. J. Comp. Neurol. 221, 358-370

Hsu, S.-M., Raine, L. and Fauger, H. (1981) Use of avidin-biotin peroxidase complex $(A B C)$ in immunoperoxidase technique: $\mathrm{A}$ comparison between $\mathrm{ABC}$ and unlabeled antibody (PAP) procedure. J. Histochem. Cytochem. 29, 577-580.

Hultcrantz, E., Linder, J. and Angelborg, C. (1977) Sympathetic effects on cochlear blood flow at different blood pressure levels. INSERM 68, 271-278.

Hultcrantz, E., Nuttall, A., Brown, M.C. and Lawrence, M. (1982) The effect of cervical synpathectomy on cochlear electrophysiology, Acta Oto-Laryngol. 94, 439-444.

Hultcrantz, E., Thorne, P. and Nuttall. A. (1986) The influence of cervical sympathetic stimulation on laser-Doppler blood flow measurements from the cochlea. XXIII Workshop on Inner Ear Biol. Abstr., p. 43.

Klinke. R. (1986) Neurotransmission in the inner ear. Hear. Res. 22, 235-243.

Klinke, R. and Evans, E.F. (1977) Fvidence that catechola- 
mines are not the afferent transmitter in the cochlea. Exp. Brain Res. 28, 315-324.

Krejci, F. and Bornschein, H. (1954) The cochlear microphonic potentials during sympathetic stimulation. Acta OtoLaryngol, 44, 154-156.

Lee, A.H. and Møller, A.R. (1985) Effects of sympathetic stimulation on the round window compound action potential in the rat. Hear. Res. 19, 127-134.

Levitt, M., Spector, S., Sjoerdsma, A. and Udenfriend, S. (1965) Elucidation of the rate limiting in norepinephrine biosynthesis in the perfused guinea pig heart. J. Pharm. Exp. Ther. 148, 1-8.

Muchnik, C., Hildesheimer, M., Nebel, L. and Rubinstein, M. (1983) Influence of catecholamines on cochlear action potentials. Arch. Otolaryngol. 109, 530-532.

Pickel, V.M., Joh, T.H. and Reis, D.J. (1975) Immunohistochemical localization of tyrosine hydroxylase in brain by light and electron microscopy. Brain Res. 85, 295-300.

Pickles, J.O. (1979) An investigation of sympathetic effects on hearing. Acta Oto-Laryngol. 87, 69-71.

Ross, M. (1971) Flourescence and electron microscopic observations of the general visceral efferent innervation of the inner ear. Acta Oto-Laryngol. Suppl. 286, 1-18.
Seymour, J.C, and Tappan, J.W. (1951) The effect of sympathetic stimulation upon the cochlear microphonic potentials. J. Laryngol. Otol. 61, 423-434.

Spoendlin, H. and Lichtensteiger, W. (1966) The adrenergic innervation of the labyrinth. Acta Oto-Laryngol. 61 . 423-434.

Suga, F. and Snow. F. (1969) Adrenergic control of cochlear blood flow. Ann. Otol, Rhinol. Laryngol. 78, 358-374.

Terayama, Y., Holz, E. and Beck, C. (1966) Adrenergic innervation of the cochlea. Ann. Otol. Rhinol. Laryngol. 99. 513-524.

Vincent, S.R. (1986) Tyrosine hydroxylase immunoreactive neurons are present in weird places in the hamster brain. Soc. Neuro Sci. Abstr. 12, 139.

Vinnikov, J.A., Govyrin, V.A., Leontieva, G.R. and Anichin, V.F. (1966) The adrenergic innervation of the organ of Corti. Dokl. Akad. Nauk. SSR 171, 484-486.

Warr, W.B., Guinan, J.J., Jr. and White, J.S. (1986) Organization of efferent fibers: The lateral and medial olivocochlear systems. In: Neurobiology of Hearing: The Cochlea, pp. 333-348. Editors: Altschuler, R.A., Hoffman, D.W. and Bobbin, R.P.. Raven Press, NY. 